\title{
Fourier-space entanglement of spin chains
}

\author{
Miguel Ibáñez-Berganza \\ INFN-Gruppo Collegato di Parma, via G.P. Usberti, 7/A, 43124, Parma, Italy \\ Dipartimento di Fisica, Università di Roma "La Sapienza", Piazzale Aldo Moro 5, 00185, Roma, Italy \\ Javier Rodríguez-Laguna \\ Dept. of Fundamental Physics, Universidad Nacional de Educación a Distancia (UNED), Madrid, Spain \\ Germán Sierra \\ Instituto de Física Teórica (IFT), UAM-CSIC, Madrid, Spain
}

(Dated: February 12, 2016)

\begin{abstract}
Entanglement between different regions in momentum space is studied for ground states of some spin-chain Hamiltonians: the XY model, the Ising model in a transverse field (ITF) and the XXZ models. In the XY and ITF cases, entanglement only takes place between states with opposite momenta. Thus, an anisotropy in the interaction induces entanglement in the momentum pairs. In the ITF case, the ferromagnetic phase is characterized by a total entropy between left- and rightmoving modes which is independent on the external field. This result characterizes the Ising phase transition in momentum space. In the critical XXZ case, we provide evidence that the maximal entropy between energy modes around the Fermi point grows logarithmically with the system size, with a prefactor which depends on the compactification radius. The slow growth of the entanglement in Fourier space with the system size provides an explanation for the success of the renormalization techniques in momentum space.
\end{abstract}

\section{INTRODUCTION}

Entanglement entropy (EE) and entanglement spectra (ES) are concepts in quantum information theory which have provided invaluable insight for quantum phase transitions and topological phases [1,3]. Area laws [4, 5] have been put forward for the ground state (GS) of many local Hamiltonians, stating that the EE of a block in real space is proportional to the number of links which must be broken in order to isolate the subsystem. A rigorous proof can be given in few situations, such as 1D gapped systems [6]. The EE for $1 \mathrm{D}$ critical states is known to receive a logarithmic correction proportional to the central charge of the associated conformal field theory (CFT) [7, 9]. The fact that entanglement is a resource for quantum computation [10] can be reversed, to state that it is the limiting feature for many numerical methods designed to study quantum many-body problems [11], such as the density matrix renormalizartion group (DMRG) [8].

Most studies of entanglement are performed in real space. Yet, many analytical and numerical methods for quantum many-body systems rely on a Fourier space description. Real space sites and momentum sites constitute two different tensor structures of the same manybody Hilbert space, thus allowing for very different entanglement patterns. The success of DMRG in momentum space [13] and the relation between momentumspace entanglement and renormalizability [12] suggest that the dependence of the maximum entanglement with the system size could be lower in momentum space than in real space for many systems of interest. A duality between entanglement in real and momentum space was uncovered in [14, 16], which applies to free fermionic sys- tems, but no general rule has been derived. Thus, while 17] reports that the XXZ critical region does not have any signatures in the ES or the EE, [18] shows that the ES in energy space can provide a unified picture of the phase diagram for $p$-wave superconductors, and [19] finds similar traces of the transition of the extended Hubbard model studying multipartite entanglement in momentum space.

For a homogeneous system, the entanglement properties of a spatial block should only depend on the block size. Fourier space, on the other hand, is inherently inhomogeneous. For systems with a Fermi surface structure, the Fermi momentum $k_{F}$ is specially relevant. Thus, it makes special sense to study the entanglement between blocks around $k_{F}$ and their complementary, and the entanglement between levels below and above $k_{F}[16$, which has proved valuable in the study of superconducting systems [15, 18]. As we will see, it is thus crucial to know the nature of our physical problem before deciding which blocks might provide a deeper insight.

This article is organized as follows. A general discussion about the meaning of entanglement in Fourier space is done in section II. Then, section III shows the behaviour of the entanglement in momentum space for the (generalized) XY model, computed analytically via a Jordan-Wigner transformation. Section [V] is devoted to the (numerical) study of the entanglement in Fourier space of the XXZ model. The article ends with a section on conclusions and proposals for further work. 


\section{ENTANGLEMENT FROM REAL TO MOMENTUM SPACE}

Let us consider a system of spinless fermions. Its Hilbert space is the tensor product of $N$ qubit Hilbert spaces: $\mathcal{H}=\mathbb{C}^{2} \otimes \cdots \otimes \mathbb{C}^{2}$. A basis can be written using the local creation operators $c_{i}^{\dagger}$ :

$$
\left|n_{1} \cdots n_{N}\right\rangle=\prod_{i=1}^{N}\left(c_{i}^{\dagger}\right)^{n_{i}}|0\rangle,
$$

where $n_{i} \in\{0,1\}$. For any single-body unitary transformation $U$, we can define a new set of creation operators: $b_{j}^{\dagger}=\sum_{i} U_{j i} c_{i}^{\dagger}$. Now we can define a new basis:

$$
\left|m_{1} \cdots m_{N}\right\rangle=\prod_{j=1}^{N}\left(b_{j}^{\dagger}\right)^{m_{j}}|0\rangle,
$$

with $m_{i} \in\{0,1\}$. Defining $\vec{n} \equiv\left\{n_{1}, \cdots, n_{N}\right\}$ and $\vec{m} \equiv$ $\left\{m_{1}, \cdots, m_{N}\right\}$, any pure state of $\mathcal{H}$ can be expressed in both bases:

$$
|\Psi\rangle=\sum_{\vec{n}} C_{\vec{n}}\left|n_{1} \cdots n_{N}\right\rangle=\sum_{\vec{m}} B_{\vec{m}}\left|m_{1} \cdots m_{N}\right\rangle .
$$

And we can write a change of basis matrix:

$$
B_{\vec{m}}=\sum_{\vec{n}} \Omega_{\vec{m}, \vec{n}} C_{\vec{n}},
$$

where $\Omega_{\vec{m}, \vec{n}}=\left\langle m_{1} \cdots m_{N} \mid n_{1} \cdots n_{N}\right\rangle$. In the particular case of fermionic states, both basis states are Slater determinants, so the scalar products can be computed efficiently:

$$
\Omega_{\vec{m}, \vec{n}}=\operatorname{det}\left(U_{\vec{m}, \vec{n}}\right),
$$

where we use the notation $U_{\vec{m}, \vec{n}}$ to be the (sub-)matrix where we only pick the rows given by the values $m_{j}=1$ and the columns with $n_{i}=1$.

When the single-body unitary matrix $U$ implements the Fourier transform, then the coefficients $B_{\vec{m}}$ are read as the Fourier expansion of the many-body wavefunction, which correspond to a different slicing of the Hilbert space as a tensor product: each qubit space corresponds now to a momentum site instead of a real space site. Each index $j$ corresponds to a certain momentum $k_{j}$, determined by the size of the system and the boundary conditions. We will focus on antiperiodic boundary conditions with even $N$. Thus, we have $k=(2 p+1) \pi / N$, with integer $p$. This way, $k=0$ and $k=\pi$ are never allowed. We always define our momenta to lie in $(-\pi, \pi]$.

If a quantum single-body state is translationally invariant, it must have a well defined momentum. A translationally invariant quantum many-body state fulfills a more involved constraint: its non-zero amplitudes $B_{\vec{m}}$ all have $\sum_{j} m_{j} k_{j}=0 \bmod 2 \pi$.

Momentum space is inherently non-homogeneous, even if real space can be considered to be so. When we study entanglement in real space, typically we are only interested in the size of the block. But in momentum space, we are also interested in the position of those blocks. Thus, we have defined a few interesting possibilities, motivated by the physics of the problems that we will study, depicted in Fig. 1
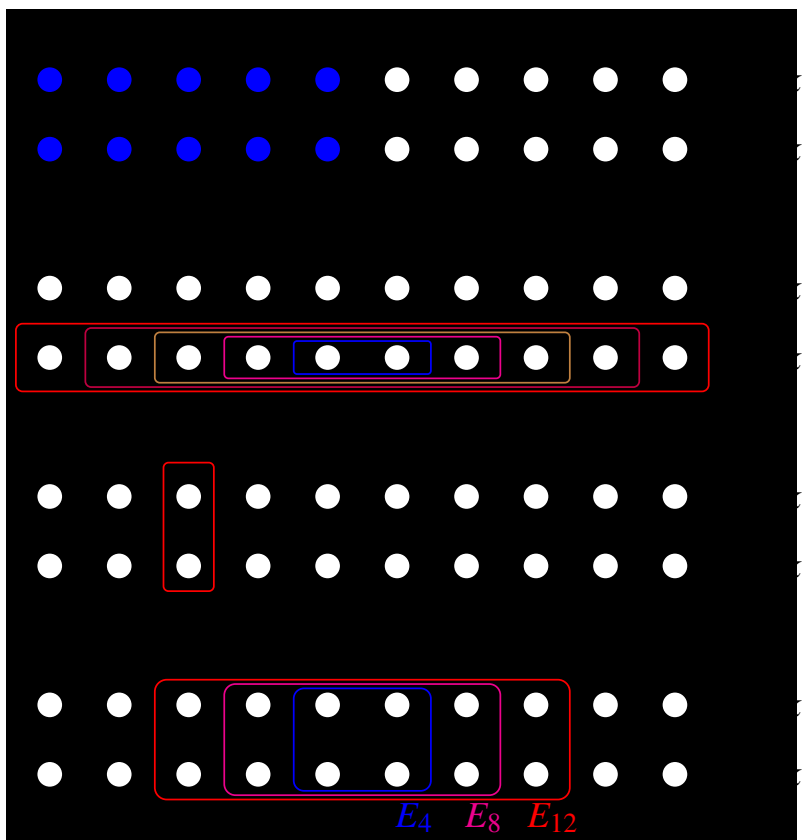

FIG. 1. Illustration of the different blocks used to study the entanglement entropy in momentum space. (A) momentum space structure, showing in blue the momenta occupied by a Fermi sea. (B) Positive momenta blocks, $P_{n}$ contains the $n$ momenta closest to the positive Fermi level, $+k_{F}$. (C) Pairing of opposite momenta, block $p_{k}$ contains the momenta $k$ and $-k$. (D) Energy blocks, $E_{n}$ contains the $n$ momenta which are closest to the Fermi energy.

Let us start our description with a Fermi sea, as depicted in Fig. 1 (A), where the Fermi momentum $\pm k_{F}$ is marked. The first type of blocks to be considered is $P_{n}$, which contains $n$ positive momenta around the positive Fermi point, see Fig. 1 (B). Thus, $P_{N / 2}$ corresponds to the block which contains all positive momenta, which we will denote simply by $P$. Another natural set of blocks can be formed by choosing the $(-k, k)$ pairs with the same energy, which we will call $p_{k}$, as shown in Fig. 1 (C). Following [18], we can also consider blocks in energy space, assuming a relation $E \sim|k|$, as in Fig. 1 (D), where $E_{n}$ contains the $n$ momenta whose energy are closest to the Fermi energy. 


\section{GENERALIZED XY MODEL}

In this section we will present our first physical system, the 1D XY model, and map it into a model of spinless fermions. The XY Hamiltonian in a one-dimensional lattice of $N$ sites and periodic boundary conditions can be written as:

$$
H=\frac{-1}{2} \sum_{j=1}^{N} \frac{1}{2}\left[(1+\gamma) \sigma_{j}^{x} \sigma_{j+1}^{x}+(1-\gamma) \sigma_{j}^{y} \sigma_{j+1}^{y}\right]+J \sigma_{j}^{z}
$$

This system is exactly solvable [20]. The procedure is to perform a Jordan-Wigner transformation, which will bring it to a fermionic Hamiltonian without particle preservation, followed by a Fourier transform of the resulting fermionic operators. One obtains the following Hamiltonian:

$$
\mathcal{H}=\sum_{j \in \Omega} A_{j} d_{j}^{\dagger} d_{j}+i \frac{B_{j}}{2}\left[d_{j}^{\dagger} d_{-j}^{\dagger}+d_{j} d_{-j}\right]
$$

where we defined $A_{j}, B_{j}$ :

$$
A_{j}=J-\cos k_{j}, \quad B_{j}=-\gamma \sin k_{j} .
$$

and where $k_{j}=2 \pi j / N$, and the set of allowed momenta is $\Omega=\left\{k_{j}\right\}_{j=-N / 2}^{N / 2}$. The operators $d_{j}$ are Fourier-space fermionic operators annihilating a fermion with momentum $k_{j}$. In the fermionic formulation, the total $z$-axis magnetization $M^{(z)}=\sum_{j}\left\langle\sigma_{j}^{z}\right\rangle$ of the spin model is related with the number of fermions, $n_{\mathrm{f}}=\sum_{j}\left\langle d_{j}^{\dagger} d_{j}\right\rangle$ as: $2 n_{\mathrm{f}}-N=M^{(z)}$. The following step is a Bogoliubov transformation of the form

$$
b_{j}^{\dagger}=u_{j} d_{j}^{\dagger}+\imath v_{j} d_{-j},
$$

with $u_{j}^{2}+v_{j}^{2}=1$ and

$$
\begin{array}{r}
u_{j}^{2}-v_{j}^{2}=A_{j} / E_{j}, \\
2 u_{j} v_{j}=B_{j} / E_{j},
\end{array}
$$

where $E_{j}$ is the energy of the $j$-th free mode, satisfying $E_{j}^{2}=A_{j}^{2}+B_{j}^{2}$. Thus,

$$
u_{j}^{2}=\frac{1}{2}\left(1+\frac{A_{j}}{E_{j}}\right), \quad v_{j}^{2}=\frac{1}{2}\left(1-\frac{A_{j}}{E_{j}}\right) .
$$

Such a transformation brings the Hamiltonian into a free Hamiltonian in the $b$-modes:

$$
\mathcal{H}=\sum_{j}\left|E_{j}\right| b_{j}^{\dagger} b_{j}-\frac{1}{2} \sum_{j} E_{j}
$$

For each eigenstate of the XY Hamiltonian (6), there exists a corresponding eigenstate of (11) with equal energy, of the form:

$$
|\theta\rangle=\prod_{j}\left[\theta_{j} b_{j}^{\dagger}+\left(1-\theta_{j}\right)\right]|\mathrm{g}\rangle,
$$

where $|\mathrm{g}\rangle$ is annihilated by all $b_{j}$ 's, and the state is characterized by the set of occupations $\theta_{j}=0,1$ in terms of $b$-modes: $\theta_{j}=\left\langle\theta\left|b_{j}^{\dagger} b_{j}\right| \theta\right\rangle$. The Hamiltonian ground state $|\mathrm{g}\rangle$ is related with the $d$ 's zero in the following form:

$$
|\mathrm{g}\rangle=\prod_{j} b_{j}|0\rangle
$$

Taking $\gamma=0$ (XX model), the Hamiltonian (7) is already diagonal in the $d$-modes, so each $d_{j}$ is correlated only with itself, $\left\langle d_{j}^{\dagger} d_{j^{\prime}}\right\rangle=\kappa_{j} \delta_{j, j^{\prime}}$, where $\kappa_{j}=0,1$ is the occupation of site $j$, and where the expected value $\langle\cdot\rangle$ is taken with respect to the $d$ operator vacuum, $|0\rangle$. In other words, the eigenstates of the Hamiltonian are product states in momentum space, hence presenting a vanishing entanglement entropy in momentum space. For $\gamma \neq 0$, however, the eigenstates (12) are such that moment $k$ is only entangled to moment $-k$. Let us call $(k,-k)$ a momentum pair. Given any block $B$ in momentum space, we need only to consider its broken pairs: if both $k$ and $-k$ belong to $B$, then they do not contribute to entanglement. Thus, without any loss of generality. let us only consider in the following blocks composed of positive momenta, see Fig. 1].

The von Neumann and Rényi entropies quantifying the amount of entanglement between $B$ and its complementary can be exactly computed throught the fermionic correlators $\left\langle d_{i}^{\dagger} d_{j}\right\rangle$ of the model. Consider the $\ell \times \ell$ reduced correlation matrix, $\mathcal{C}$ :

$$
\mathcal{C}_{j, j^{\prime}}=\left\langle\theta\left|d_{j}^{\dagger} d_{j^{\prime}}\right| \theta\right\rangle \quad k_{j}, k_{j^{\prime}} \in \mathcal{A}_{\ell} .
$$

Using the inverse Bogoliubov transformation (9), it is found to be

$$
\begin{array}{r}
\mathcal{C}_{i, j}=\delta_{i, j} \lambda_{j}, \\
\lambda_{j} \equiv\left[u_{j}^{2} \theta_{j}+v_{j}^{2}\left(1-\theta_{-j}\right)\right] .
\end{array}
$$

Let us denote by $\rho_{B}$ the reduced density matrix of the state (12) in $B$, i.e., with the degrees of freedom of the complementary of $B$ traced out. It is a tensor product of $2 \times 2$ reduced density matrices in different sites of $B$ : $\rho_{B}=\bigotimes_{j \in B} \varrho_{j}$. The entropy of entanglement is obtained from the eigenvalues of $\varrho_{j},\left\{\lambda_{j}, 1-\lambda_{j}\right\}$, being $\lambda_{j}$ the eigenvalue of the $j$-site reduced correlation matrix (15). The von Neumann entropy is given by

$$
S\left[\rho_{B}\right]=\sum_{k_{j} \in B} H_{2}\left(\lambda_{j}\right)
$$


where $H_{2}(x) \equiv-(x \log x+(1-x) \log (1-x))$. Equivalently, the Rényi entropy of order $\alpha>1$ reads:

$$
S_{\alpha}\left[\rho_{B}\right]=\frac{1}{1-\alpha} \sum_{k_{j} \in B} \log \left[\lambda_{j}^{\alpha}+\left(1-\lambda_{j}\right)^{\alpha}\right] .
$$

Alternatively, one can obtain Eqs. (16) and (17) computing explicitly the reduced $j$-site correlation matrix using Eq. (13); in the $\theta_{j}=0$ case, for instance, it is simply: $\varrho_{j}=v_{j}^{2} d_{j}^{\dagger}|0\rangle_{j}\left\langle 0\left|d_{j}+u_{j}^{2}\right| 0\right\rangle_{j}\langle 0|$. Assuming even states $\theta_{j}=\theta_{-j}$, the entropies do not depend on the state $\theta$ :

$$
\begin{aligned}
S\left[\rho_{B}\right] & =\sum_{k_{j} \in B} H_{2}\left(u_{j}^{2}\right), \\
S_{\alpha}\left[\rho_{B}\right] & =\frac{1}{1-\alpha} \sum_{k_{j} \in B} \log \left[u_{j}^{2 \alpha}+v_{j}^{2 \alpha}\right] \quad \alpha>1 .
\end{aligned}
$$

Our preferred blocks to show the structure of the entanglement in the XY model will be the positive momentum blocks centered on the Fermi point, as illustrated in 1(B), i.e., the $P_{n}$ blocks.

\section{A. XY model with $J=0$}

Let us illustrate the laws (18) in their particular realizations for the XY model ground states. Our first example is the $J=0$ case. The model with $J=0$ is gapped except for the point $\gamma=0$ which corresponds to the XX model, whose ground state is a product state in momentum space. The $\gamma$ parameter may be regarded as a mass term. For $\gamma=0$, the state is

$$
|\mathrm{g}\rangle=\prod_{\left|k_{j}\right|<\pi / 2} d_{j}^{\dagger}|0\rangle .
$$

The diagonal of matrix $\mathcal{C}$ in Eq. (15) gives the occupations of $d$ modes in the ground state, $\left\langle\mathrm{g}\left|d_{j}^{\dagger} d_{j}\right| \mathrm{g}\right\rangle=v_{j}^{2}$, which are presented in Fig. 2 (A).

We also present the entanglement entropy of the single momentum states, $S\left[\varrho_{j}\right]$, which are given by each one of the terms in the sum of Eqs. 116, 17). Fig. 2 (B) shows $S\left[\varrho_{j}\right]$ for $j=0, \ldots, N / 2$ as a function of both $k_{j}$ and $\gamma$. While for small values of $\gamma$ only those momenta immediately around the Fermi point at $k_{F}=\pi / 2$ present a significant amount of entanglement, as $\gamma$ becomes larger and larger the distribution of entanglement $S\left[\varrho_{j}\right]$ as a function of $k_{j}-k_{F}$ becomes broader.

In Fig. $2(\mathrm{C})$, we present the $J=0$ entropy $S\left(P_{n}\right)$ as a function of $n=0, \ldots, N / 2$ for 11 different values of $\gamma$ from 0 to 1.4 and $N=100$. For $\gamma=0$ one also observes a vanishing entropy for all $n$, as already mentioned. Let us remark that the critical case presents the minimum momentum space entropy for all $n$, as opposed to the behaviour of the real-space entanglement entropy. For
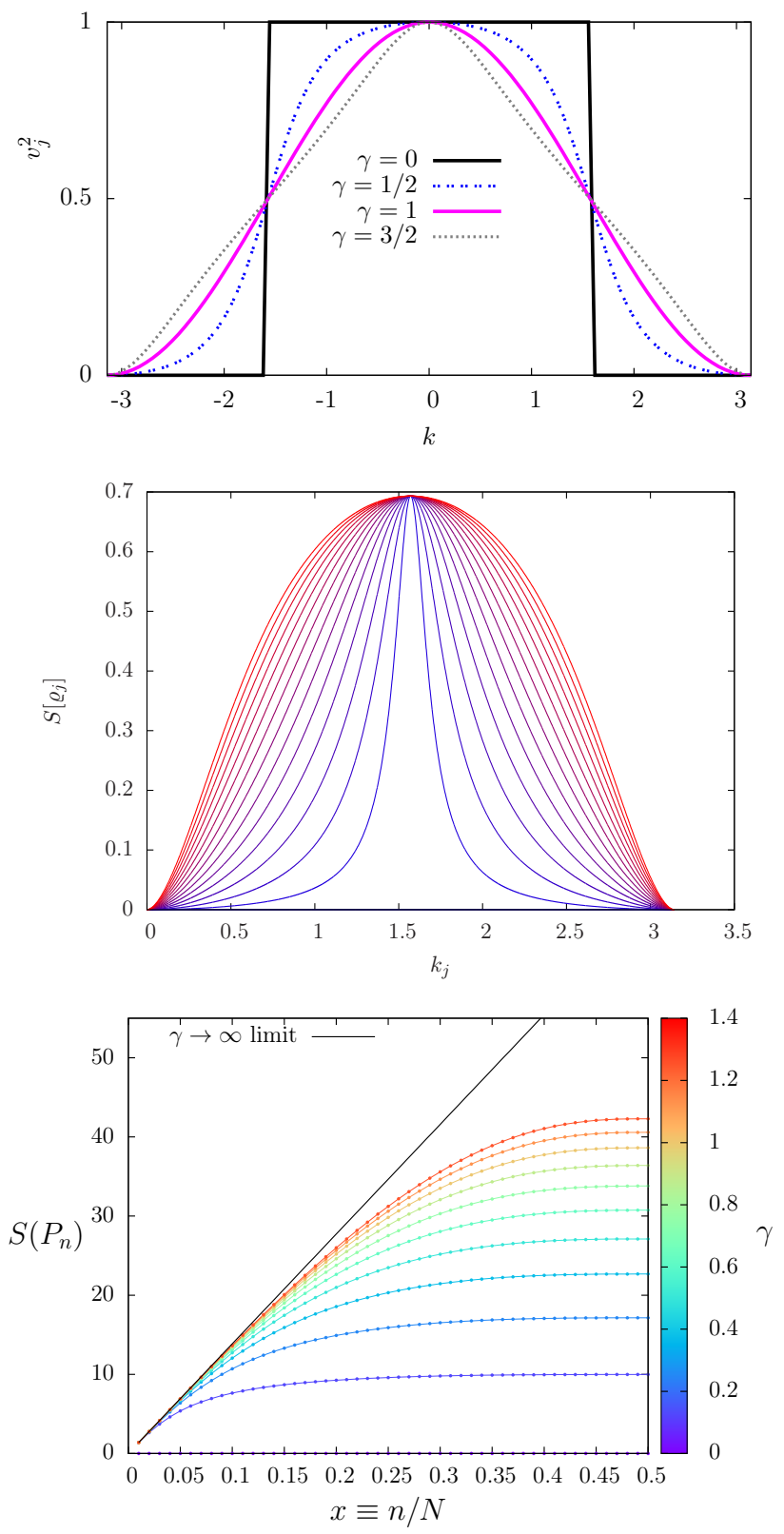

FIG. 2. Entanglement in momentum space for the XY model of Eq. (6) with $J=0$, for different values of $\gamma$. (A) Occupations of the different modes, $\left\langle\mathrm{g}\left|d_{j}^{\dagger} d_{j}\right| \mathrm{g}\right\rangle=v_{j}^{2}$ vs. $k_{j}$. (B) Contribution of each mode $k$ to the entanglement entropy, $S\left[\varrho_{j}\right]$, with $\gamma$ ranging from 0 (identically zero) to 1.5 (red). (C) Entanglement entropy of positive momentum blocks $S\left(P_{n}\right)$ as a function of the block size $x=n / N$ with $N=100$, along with the $\gamma \rightarrow \infty$ limit, $S\left(P_{n}\right)=n \log 2$. Again, for $\gamma=0$ the entropy is identically zero.

increasing $\gamma$ we get increasing entropy. The $\gamma \rightarrow \infty$ case presents a linear growth of the entropy with the system size, $S\left(P_{n}\right) \rightarrow n \log 2$, as can be analytically proved from the exact solution.

Notice that, for $\gamma \rightarrow \infty$, each pair $(-k, k)$ becomes 
maximally entangled, contributing $\log (2)$ to the entropy of the block of positive momenta. Thus, the entanglement entropy of the block $P_{N / 2}$ tends to its maximal possible value, $N \log (2) / 2$. Although it is tempting to think of this state as a highly entangled state, we should take into account that it depends on the neighborhood structure that we impose on momentum space. If we consider $k$ to be neighbor to $-k$, then the entanglement is only of short range.

\section{B. Ising model}

Let us now study the Ising model, Eq. (6) with $\gamma=1$, for different values of $J$. The system is gapped for all $J \neq$ 1 , and for $J=1$ it presents a quantum phase transition. The $J<1$ phase is ferromagnetic, while for $J>1$ the system is paramagnetic.

The ground state occupations $\left\langle d_{j}^{\dagger} d_{j}\right\rangle$ are shown in Fig. 3 (A). As opposed to the previous case, the number of fermions $n_{\mathrm{f}}$ (i.e., the sum of $v_{j}^{2}$ for all $j$ ) is not constant, but decreases with $J$, as illustrated in Fig. 33 (A, inset). This fact indicates that the blocks $P_{n}$ defined in Fig. 1 have lost its special relevance, since the Fermi point is no longer defined independently of $J$. However, we will concentrate on the entanglement of individual momentum pairs, $S\left[\varrho_{j}\right]$, and the total entanglement of positive versus negative momenta, $P_{N / 2}$.

Fig. 3(B) shows the entanglement entropy contributed by each pair $\left(k_{j},-k_{j}\right), S\left[\varrho_{j}\right]$, for different values of $J$, given by

$$
s(k, J)=H_{2}\left(\frac{1}{2}+\frac{J-\cos k}{2 \sqrt{(J-\cos k)^{2}+\sin ^{2} k}}\right)
$$

The value $J=0$ corresponds to the blue centered line. Let $k_{c}(J)$ denote the momentum for which $s\left(k_{c}, J\right)$ attains is maximum. For $J \leq 1, k_{c}=\arccos (J)$, and $s\left(k_{c}, J\right)=\log 2$, i.e. that mode is maximally entangled. For $J=1, k_{c}=0$, the maximally entangled mode is the zero mode. For $J>1$, in the paramagnetic phase, the maximal value $\max _{k} s(k, J)$ decreases with $J$ and $k_{c}$ increases again.

In Fig. 4 (A) we show the entropy of finite blocks around the Fermi point, $S\left(P_{n}, J\right)$ (check Fig. 11), as a function of $x=n / N$ for different values of $J$ in a finite system with $N=200$. For $J=0$ the entropy is maximal for all $x$, see the top blue curve in Fig. 3 (C). The curve becomes a straight line for the critical value, $J=1$. Notice that, for all $J \leq 1$ the $x=1 / 2$ entropy is constant. In other terms, along the ferromagnetic phase, the entanglement between the positive and negative momenta is independent of $J$. This value, $S\left(P_{N / 2}, J\right)$, is given by the expression
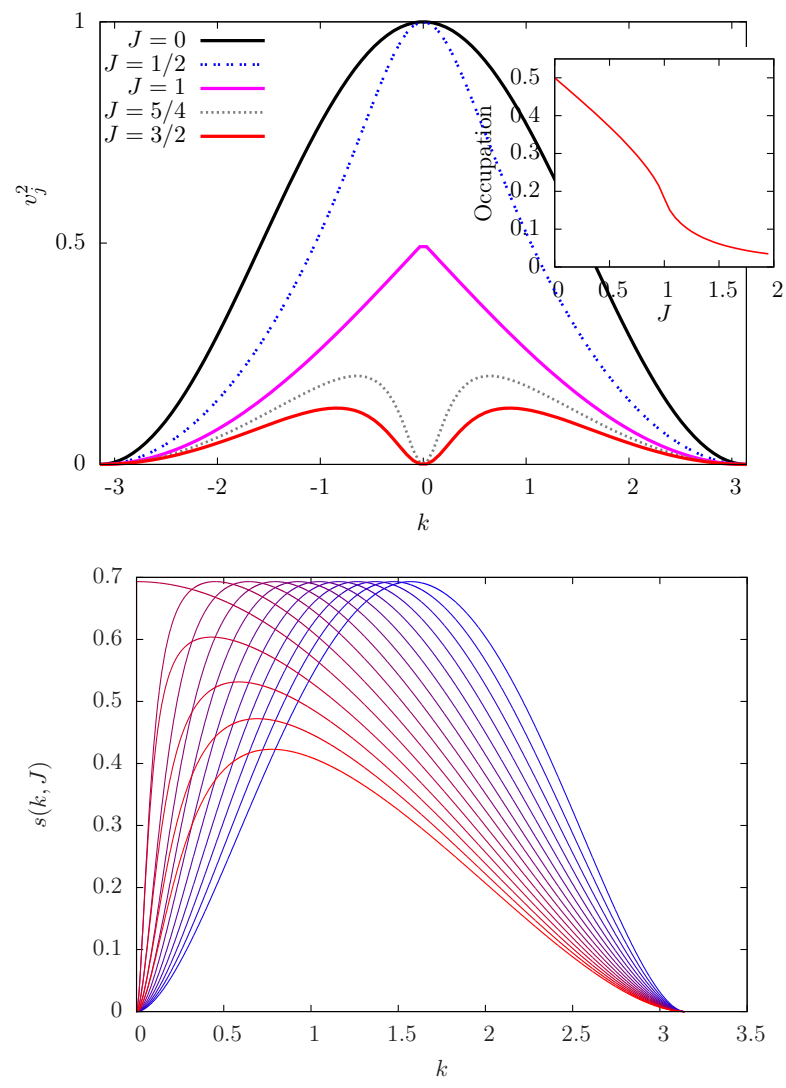

FIG. 3. Entanglement in momentum space of the Ising model in a transverse field (ITF) defined in (6) for $\gamma=1$. (A) Occupations of the different modes, $v_{j}^{2}$ vs. $k_{j}$. Inset: total number of particles, as a function of $J$. Notice the larger slope for $J=1$. (B) Contribution of each mode $k$ to the entanglement entropy, $s(k, J)=S\left[\varrho_{j}\right]$ for different values of $J$. The blue line, $J=0$, is symmetrical with respect to $k_{c}=\pi / 2$. The momentum of maximal entropy, $k_{c}=\arccos J$ decreases with $J$ until, for $J=1$, it reaches zero. The maximal entropy contribution $s\left(k_{c}(J), J\right)=\log 2$ for $J \leq 1$. For $J>1$ (red lines), the maximal entropy is lower than $\log 2$.

$$
S\left(P_{N / 2}, J\right)=\sum_{0<k_{j}<\pi} H_{2}\left(u_{j}^{2}(J)\right) \approx \frac{N}{\pi} \int_{0}^{\pi} d k s(k, J)
$$

where the last step is taken in the thermodynamic limit, and corresponds to the area under each of the curves of Fig. 3 (B). The area under all these curves is equal for $J \leq 1$, and the following result can be proved:

$s_{0} \equiv \lim _{N \rightarrow \infty} \frac{S\left(P_{N / 2}, J\right)}{N}=\log 2-1 / 2 \approx 0.193 \quad$ if $J \leq 1$

The point at which it is simplest to evaluate the integral is at $J=0$, where it becomes: 


$$
s_{0}=\frac{1}{\pi} \int_{0}^{\pi / 2} d \phi H_{2}\left(\cos ^{2}(\phi)\right)
$$

Fig. 4 (B) shows the value of the entropy per site between the positive and negative momenta, $s_{N}(J) \equiv$ $S\left(P_{N / 2}, J\right) / N$ for some finite-size values (dots) and the thermodynamical limit (continuous black line), where we can see that it stays constant for $J \leq 1$ and decays linearly shortly after $J>1$. The derivative is, therefore, discontinuous at that point.
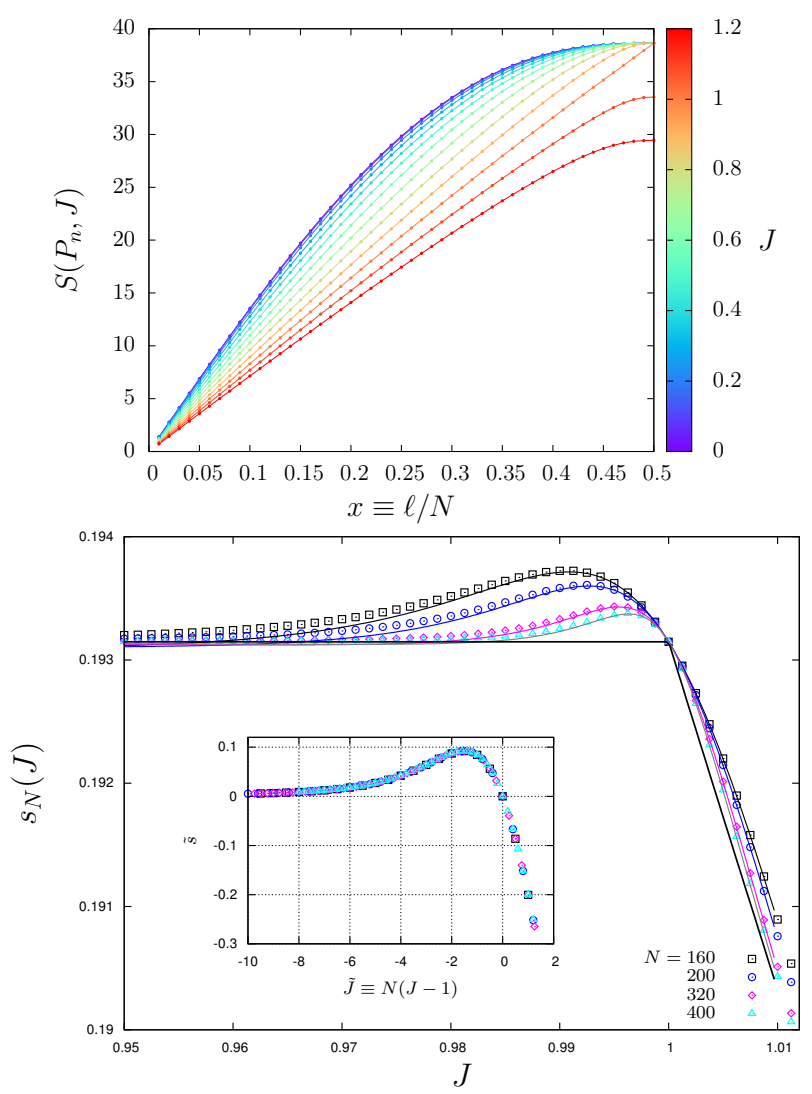

FIG. 4. Entanglement of blocks of positive momenta $P_{n}$ ITF model, Eq. (6) with $\gamma=1$. (A) Entropy of the $P_{n}$ block, $S\left(P_{n}\right)$, as a function of the block size fraction, $x=n / N$, for different values of $J$ for $N=200$. Notice that the maximal value of $S\left(P_{n}\right)$ remains constant for all $J \leq 1$, and decreases for $J>1$. (B) Entanglement between positive and negative momenta for the ITF model per site, $s_{N}(J)=S\left(P_{N / 2}, J\right) / N$, for different sizes. Notice how, in the thermodynamic limit (continuous black line), the entanglement is constant for $J \leq 1$ and decays linearly for $J>1$. Moreover, the colored continuous lines are given by approximation (25), which singles out the lowest mode. Inset: collapse of the finite-size curves, $\tilde{s}(\tilde{J})$, see Eq. (24).

The finite-size entropies shown by the dots in Fig. 4 (B) provide very relevant information. They all follow the scaling form

$$
s_{N}(J) \approx s_{0}+\tilde{s}(\tilde{J})
$$

where $\tilde{J}=N(J-1)$, which can be understood as a scaling variable since $J-1$ is the inverse of a correlation length. The inset of Fig. 4 (B) shows the collapse of the $\tilde{s}=$ $s_{N}(J)-s_{0}$ curves, when expressed as a function of $\tilde{J}$, in both phases. The origin of this $\tilde{s}(\tilde{J})$ scaling lies in the fact that the deviation from the continuum limit is due mostly to the the smallest momentum, $\Lambda_{N}=\pi / N$. If we single out its contribution from the sum, as it is customary in the study of Bose-Einstein condensation, we obtain the approximation

$$
S\left(P_{N / 2}, J\right) \approx H_{2}\left(u^{2}\left(\Lambda_{N}\right)\right)+\frac{N}{2 \pi} \int_{2 \pi / N}^{\pi} d \phi H_{2}\left(u^{2}(\phi)\right),
$$

which is shown by the colored continuous lines of Fig. 4 (B). This approximation is very accurate in the vicinity of the phase transition, and we can see that the position of their maxima are precisely reproduced.

\section{ENTANGLEMENT IN THE XXZ MODEL}

Let us now investigate the entanglement entropy in momentum space of the XXZ model. Through the Jordan-Wigner transformation, this corresponds to an interacting fermionic model, which we can write as

$$
H_{X X Z}=-\frac{1}{2} \sum_{i} c_{i}^{\dagger} c_{i+1}+\text { h.c. }+\Delta \sum_{i} n_{i} n_{i+1}
$$

where $n_{i}=c_{i}^{\dagger} c_{i}$ and endowed with anti-periodic boundary conditions $(\mathrm{APBC})$, i.e., $c_{N+1}^{\dagger} \equiv-c_{1}^{\dagger}$. Notice that the number of particles is preserved in this case, so we can restrict ourselves to the case of half-filling. This model is known to be critical for $\Delta \in(-1,1]$, with central charge $c=1$. Moreover, the GS of Eq. (26) describes a Luttinger liquid 24], which is characterized by Luttinger parameter

$$
K=\frac{\pi}{2(\pi-\operatorname{acos}(\Delta))}
$$

\section{A. The Néel limit}

In the limit where $\Delta \rightarrow+\infty$ the ground state of (26) becomes a Néel state, which is a superposition of two factorized states in real space,

$$
|\Psi\rangle=\frac{1}{\sqrt{2}}(|101010 \cdots\rangle+|010101 \cdots\rangle)
$$

Let us consider the relevant momenta $\left\{k_{j}\right\}, j \in$ $\{1, \cdots, N\}$ ordered from $-\pi$ to $\pi$ and symmetrically placed around zero, so that $k_{j}=-k_{N+1-j}$. The basis states can be written as $\left|m_{1} \cdots m_{N / 2} ; m_{N / 2+1} \cdots m_{N}\right\rangle$, 
with $m_{j} \in\{0,1\}$ the occupation of the momentum $k_{j}$. Then, the Néel state can be written as

$$
|\Psi\rangle=\sum_{m_{1} \cdots m_{N / 2}} C_{m_{1} \cdots m_{N / 2}}\left|m_{1} \cdots m_{N / 2} ; \bar{m}_{N / 2} \cdots \bar{m}_{1}\right\rangle
$$

where $\bar{m}_{j}=1-m_{j}$, i.e. the occupation of level $k_{j}$ is always the opposite of level $-k_{j}$. The amplitudes $C_{m_{1} \cdots m_{N / 2}}$, disregarding normalization, are given by:

$$
C_{m_{1} \cdots m_{N / 2}}=\operatorname{Even}\left(\sum_{i=1}^{N / 2} m_{i}\right)(-1)^{\sum_{p=1}^{N / 4} m_{2 p}},
$$

where $\operatorname{Even}(n)$ is defined as 1 if $n$ is even and zero otherwise. This expression means that the non-zero wavefunction components have an even number of particles with positive (negative) momenta. Moreover, all the amplitudes are equal in absolute value, and their sign is given by the parity of the occupation of the even-indexed momenta. Notice that all modes are equally occupied, $\left\langle m_{j}\right\rangle=1 / 2$.

The entanglement structure of this state is as follows:

- The entropy of the positive momenta blocks $P_{n}$ (Fig. 1(B)), is $S\left(P_{n}\right)=n \log 2$ if $n<=N-2$.

- The block which contains all positive momenta, $P_{N / 2}$, is special, and its entropy is $S\left(P_{N / 2}\right)=$ $(N-1) \log 2$.

- All the momentum pair blocks, $p_{k}$ which contain a pair $\{k,-k\}$ (Fig. 1 (C)), have entropy $\log (2)$.

- Blocks with two momenta which do not correspond to the same energy, on the other hand, present maximal entropy $2 \log (2)$.

- Energy blocks $E_{n}$, Fig. 1 (D), also have entropy $\log (2)$.

\section{B. Entanglement in Fourier space of the the XXZ model}

We have obtained numerically the GS of Hamiltonian (26) for $N$ up to 20, and performed a numerical many-body Fourier transform of the resulting GS, as described in section [I] Due to the APBC, the set of allowed momenta come always in pairs $k_{i}= \pm i \pi / N$ and $i \in\{1,3, \cdots, N-1\}$. For $L=2 \bmod 4$, the GS is exactly degenerate for all $\Delta$, so we restrict ourselves to $N$ multiple of 4 .

First of all, we have investigated the occupation number of each momentum, in order to check the known results regarding Luttinger liquid theory. Fig. 5 shows the occupation of each $k$-mode, $n_{k} \equiv\left\langle\Psi\left|b_{k}^{\dagger} b_{k}\right| \Psi\right\rangle$ for $N=20$ and several values of $\Delta=\{0,0.2,0.4, \cdots, 1.4\}$, along with a fit to the Luttinger liquid expression

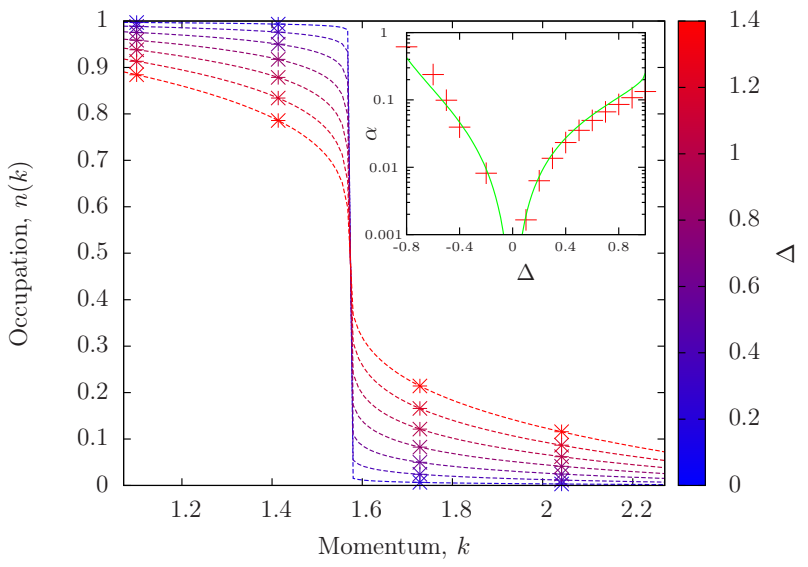

FIG. 5. Momentum space occupations for the GS of the XXZ model, obtained numerically for $N=20$ and several values of $\Delta$, and their fit to the Luttinger prediction (31). The dependence of the occupation exponent with the anisotropy $\Delta$ is shown in the inset, along with the theoretical prediction, given by Eqs. (31) complemented with (27) and (32).

$$
n_{k} \approx\left|k-k_{F}\right|^{\alpha} .
$$

The value of the exponent $\alpha$ is related to the Luttinger parameter $K$ through the following expression

$$
\alpha=\frac{1}{2}\left(K+K^{-1}\right)-1,
$$

and this last relation is checked in the inset of Fig. 5

The entropy of the positive vs negative momenta, $S\left(P_{N / 2}\right)$ (see Fig. 1) is shown in Fig. 6 (A) as a function of the system size, $N$ for several values of $\Delta$, where we show only positive values of $\Delta$ for clarity. The dependence with the system size is linear for all values of $\Delta$. The inset shows the dependence of the entropy between positive and negative modes with $\Delta$ for $N=20$. A very good fit can be made to a power law, with a different exponent for positive and negative $\Delta: S\left(P_{N / 2}\right) \sim \Delta^{1.62}$ for $\Delta>0$ and $\Delta^{1.83}$ for $\Delta<0$.

The right panel, Fig. 6 (B), shows the entropy of blocks of the form $p_{k}$ (see Fig. 1), which contain only a pair of opposite momenta, as a function of $k$, for $N=20$ and several values of $\Delta>0$. Notice that, in the XY and ITF model, all those entropies were zero. In all cases, this entropy is highest for $k \approx k_{F}$, and decays exponentially away from the Fermi point. The fits in Fig. 6 (B) are done to an expression of the form

$$
S_{k} \approx S_{k_{F}} \exp \left(-\left|k-k_{F}\right| / \sigma\right)+S_{0},
$$

where $\sigma$ provides a measure of the extent to which momenta away from the Fermi surface can be removed without alteration of the rest of the state. This $\sigma$ parameter grows as $|\Delta|$ does. 

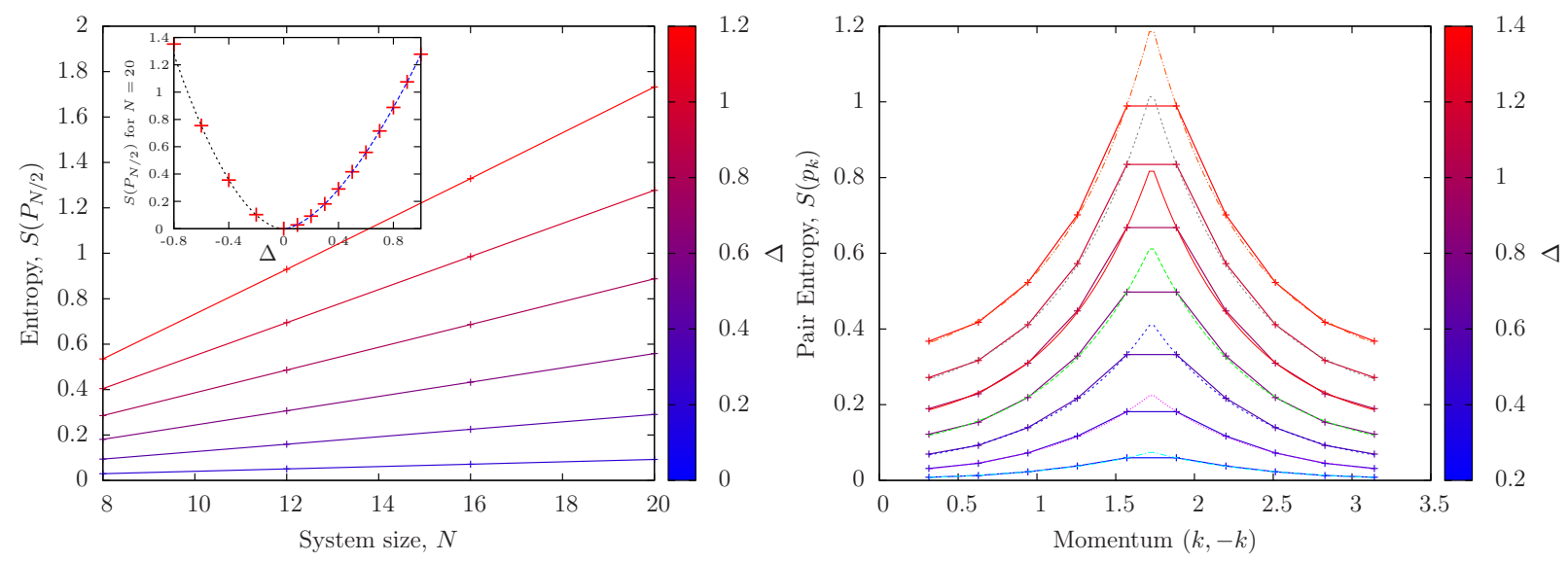

FIG. 6. (A) entropy between positive and negative momenta, $S\left(P_{N / 2}\right)$, of the GS of the XXZ model for different values of $\Delta$ and $N$ up to 20 . We only show positive values of $\Delta$ for convenience. In all cases, the plot is very accurately linear. Inset: $S\left(P_{N / 2}\right)$ with $N=20$ as a function of $\Delta$, along with the two different power-law fits, for $\Delta>0, S \sim \Delta^{1.62}$ and for $\Delta<0$, $S \sim \Delta^{1.83}$. (B) Entropy of the block containing only an opposite pair of momenta, $S\left(p_{k}\right)$ as a function of $k$, which takes the maximal value for $k \approx k_{F}$. In all cases, the entropy decays as a power-law of the distance to the Fermi momentum.

We have also studied the blocks containing the $n$ momenta which are closest to the Fermi energy, denoted by $E_{n}$ in Fig. 1. Fig. $7(\mathrm{~A})$ shows the entropy $S\left(E_{n}\right)$ as a function of $n$ for $N=20$ for different positive values of $\Delta$. This case bears the strongest similarity to the energy blocks studied previously in [18]. It can be noticed that the value $S\left(E_{n}\right)$ always decreases for large $n$, showing that separating the momenta which are further away from the Fermi surface always has a smaller entropic cost. The maximal value of $S\left(E_{n}\right), S_{\max }$ is always found for small values of $n$.

The panel (B) of Fig. 7 shows the maximal entropy $S_{\max }$ as a function of $N$ for different positive values of $\Delta$. When $\Delta$ is in the critical region, $\Delta \in(-1,1]$, the best fit is always to the form

$$
S_{\log }(N)=\Theta \log (N)+\beta+\gamma / N^{2},
$$

i.e., a logarithmic growth with a finite-size correction of $N^{-2}$. Outside the critical region, and even for $\Delta=1$, the best fit is not logarithmic, but to a power-law growth. The inset of Fig. 7 (B) shows the logarithmic growth prefactor, $\Theta$, as a function of $\Delta$. It presents a minimum, $\Theta=0$, for the XX case, $\Delta=0$, and appears to grow linearly for positive $\Delta$.

\section{CONCLUSIONS}

The structure of a many-body quantum state can be studied by slicing the Hilbert space in different ways. Traditionally, we consider the entanglement of real space blocks, but in some cases we can gather useful information using other bases, such as Fourier space. The success of DMRG in momentum space for different models points at a slow growth of entropy with the system size if the path is chosen appropriately. In this work we have studied the entanglement structure in Fourier space of spin chains, described as fermionic states through the JordanWigner transformation. We have benefitted from both analytical and numerical tools in our study.

First, we have studied the generalized XY model, which can be analytically solved using a Bogoliubov transformation. In all cases, momentum $k$ is only coupled to its conjugate $-k$. For the XX model, the wavefunction is factorizable. Introducing the $\gamma$ parameter, which measures the anisotropy between the $X$ and $Y$ axes, we couple the momenta pairs, which for $\gamma \rightarrow \infty$ become maximally entangled. The Ising model in a transverse field (ITF) can also be studied analytically. In that case, we find that the entanglement entropy per site between the positive and negative momenta is constant in the ferromagnetic phase and decreases linearly with the external field in the paramagnetic phase for $J$ close to $J=1$. Thus, there is a clear signature of the quantum phase transition. Near the critical point, the finite size corrections to the entropy can be obtained by singling out the contribution of the lowest momentum.

We have also studied the XXZ model numerically, with sizes up to $N=20$. In this case, the most salient feature is that the maximal entropy among the energy-space blocks $E_{n}$, which contain the $n$ closest momenta to the Fermi energy, grows with $\log (N)$ in the critical region. The prefactor, nonetheless, is not related to the central charge, but instead it depends on the compactification radius, which is given by the anisotropy parameter $\Delta$. Out of the critical region, the entropy of energy blocks grows faster than logarithmically, very likely as a power-law. The Néel state, which is the limit for infinite anisotropy, has also very peculiar features in Fourier space, such as a constant entropy for all of energy blocks.

Is there a complementarity relation between entanglement in real and momentum space? The question is even 

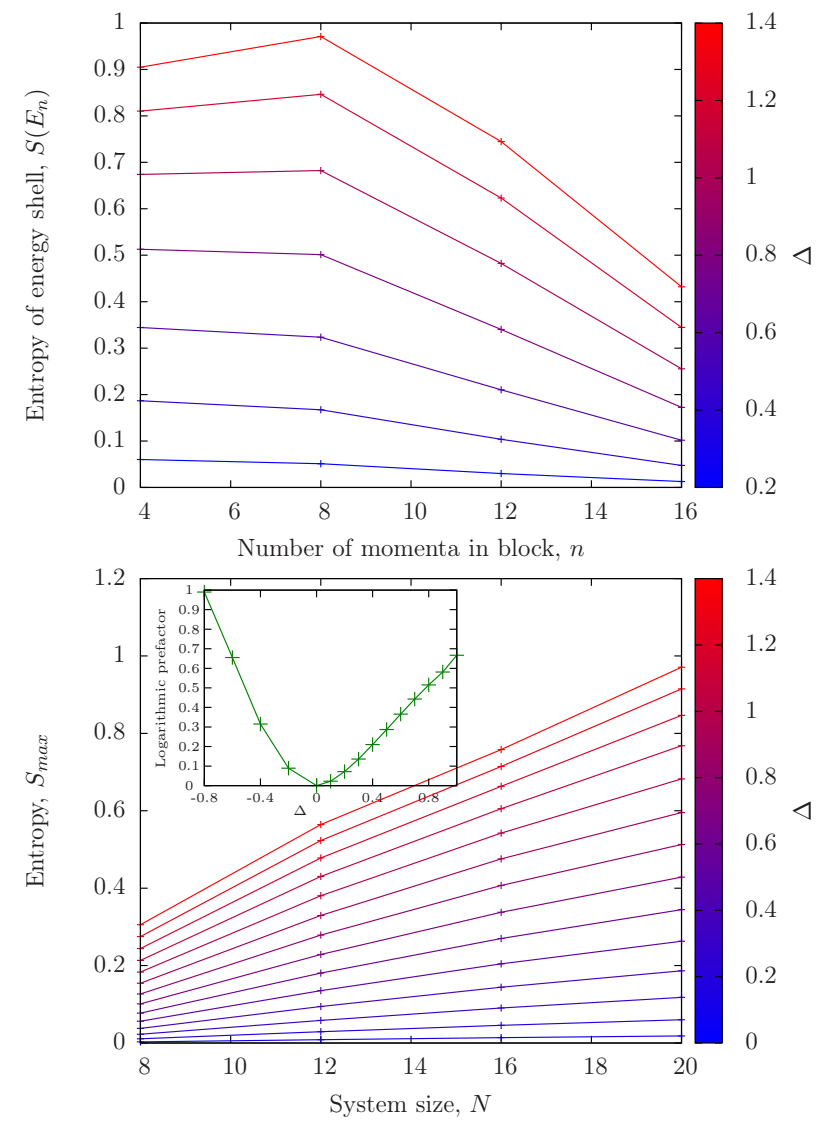

FIG. 7. (A) Entanglement entropy of blocks formed by the $n$ energy levels closest to the Fermi energy, $E_{n}$ (see Fig. 1), for different positive values of $\Delta$, also for $N=20$. (B) Maximal entanglement entropy of the $E_{n}$ blocks, $S_{\max }$ as a function of $N$, for different values of positive $\Delta$. The best fit is to a logarithmic growth, (34) with $N^{-2}$ corrections. Inset: prefactor of the logarithmic term as a function of $\Delta$, both positive and negative. In all cases, the fit error is less than $10^{-7}$ for $\Delta \in(-1,1)$.

difficult to formulate rigorously, due to the inherent inhomogeneity of momentum space. For example, which blocks should be used for the comparison? One may characterize the maximal entanglement $S_{M}$ in momentum space using a minimax definition. Let $\mathcal{P}$ be the set of all permutations of the (momentum) sites. Given $p=\left\{s_{1}, \cdots, s_{N}\right\} \in \mathcal{P}$, we may obtain the maximal entropy among the blocks starting from $s_{1}$, and define:

$$
S_{M} \equiv \min _{\mathcal{P}} \max _{m} S\left(\left\{s_{1}, \cdots, s_{m}\right\}\right)
$$

(See 25] for a similar idea in the application of the DMRG on networks). Equivalently, we can consider the minimal entropy among all blocks of a given size $m$, and maximize on $m$ :

$$
S_{M} \equiv \max _{n} \min _{|B|=n} S(B) .
$$

Both expressions must yield the same result. For example, for a 1D translation-invariant system in real space, the minimax entropy block will contain no holes, and the optimal permutation will follow the 1D structure. For the XXZ model in momentum space we conjecture that the minimax entropy takes place for the energy blocks around the Fermi surface. Thus, we propose to investigate the relation between these minimax entropies in real and momentum space.

Moreover, it is relevant to ask whether the signatures for critical behavior in Fourier space that we have discussed can be summed up into a universal criterion, at least for 1D systems. Numerical investigation of the many-body Fourier transform is very demanding computationally, thus a conceptual breakthrough is necessary at this step.

\section{ACKNOWLEDGMENTS}

We would like to thank J. Dukelsky and F. Alcaraz for useful discussions. This work was funded by grants FIS2012-33642 and FIS-2012-38866-C05-1, from the Spanish government, QUITEMAD+ S2013/ICE-2801 from the Madrid regional government and SEV-2012-0249 of the "Centro de Excelencia Severo Ochoa" Programme.
[1] L Amico, R Fazio, A Osterloh, V Vedral, Rev. Mod. Phys., 80, 517-576 (2008).

[2] H Li, FDM Haldane, Phys. Rev. Lett. 101, 010504 (2008).

[3] X Chen, Z-Ch Gu, X-G Wen, Phys. Rev. B 82, 155138 (2010).

[4] J Eisert, M Cramer, MB Plenio, Rev. Mod. Phys. 82, 277 (2010).

[5] MM Wolf, F Verstraete, MB Hastings, JI Cirac, Phys. Rev. Lett. 100, 070502 (2008).

[6] MB Hastings, J. Stat. Mech. P08024 (2007).

[7] G Vidal, JI Latorre, E Rico, A Kitaev, Phys. Rev. Lett. 90, 227902 (2003).
[8] SR White, Phys. Rev. Lett. 69, 2863 (1992).

[9] P Calabrese, J Cardy, J. Stat. Mech. P06002 (2004).

[10] MA Nielsen, IL Chuang, "Quantum computation and quantum information", Cambridge Univ. Press (2000).

[11] G Vidal, Phys. Rev. Lett. 91, 147902 (2003).

[12] V Balasubramanian, MB MacDermott, M van Raamsdonk, Phys. Rev. D 86, 045014 (2012).

[13] T Xiang, Phys. Rev. B 53, R10445 (1996).

[14] D Gioev, I Klich, Phys. Rev. Lett. 96, 100503 (2006).

[15] J Dukelsky, G Sierra, Phys. Rev. Lett. 83, 172 (1999).

[16] CH Lee, P Ye, X-L Qi, J. Stat. Mech. P10023 (2014).

[17] R Lundgren, J Blair, M Greiter, A Läuchli, GA Fiete, R Thomale, Phys. Rev. Lett. 113, 256404 (2014). 
[18] J Rodríguez-Laguna, M Ibáñez Berganza, G Sierra, Phys. Rev. B 90 041103(R) (2014).

[19] A Anfossi, P Giorda, A Montorsi, Phys. Rev. B 78, 144519 (2008).

[20] E Lieb, T Schultz, D Mattis, Ann. Phys. 16, 407 (1961).

[21] JI Latorre, E Rico, G Vidal, Q. Inf. Comp. 4, 48 (2004).

[22] M Ibáñez Berganza, F Castilho Alcaraz, G Sierra, J. Stat.
Mech. P01016 (2012).

[23] F Castilho Alcaraz, M Ibáñez Berganza, G Sierra, Phys. Rev. Lett. 106, 201601 (2011).

[24] T Giamarchi, Quantum physics in one dimension, Clarendon Press (2003).

[25] J. Rodriguez-Laguna, J. Phys. A: Math. Theor. 40, 12043 (2007). 